\title{
Investigación en la Facultad de Medicina de San Fernando: Importancia de la participación científica médico estudiantil
}

\author{
Montenegro-Idrogo, Juan José , $^{1,2, a}$, Borja-García, Rubén ${ }^{1,2, a}$, Gutiérrez, César ${ }^{1,2,3, b}$ \\ 'Facultad de Medicina San Fernando, Universidad Nacional Mayor de San Marcos (UNMSM), Lima, Perú. \\ ${ }^{2}$ Sociedad Cientifica San Fernando, Lima, Perú. \\ ${ }^{3}$ Instituto de Medicina Tropical Daniel Alcides Carrión, Facultad de Medicina, Universidad Nacional Mayor de San Marcos, Lima, Perú. \\ ${ }^{a}$ Estudiante de Medicina, ${ }^{b}$ Médico Epidemiólogo.
}

\section{Sr. Editor:}

En el número 1 del presente año de su revista, el Dr. Mendoza-Arana, a través de su artículo referido a la investigación en educación médica en la Facultad de Medicina San Fernando, menciona indicadores de productividad científica de la Facultad de Medicina sobre el tema de educación médica durante los años 2005-2010. Para ello toma como referentes de información los libros de resúmenes de los congresos de la ASPEFAM, Jornadas Científicas Sanfernandinas, además de dos revistas científicas: Anales de la Facultad de Medicina y la Revista Peruana de Medicina Experimental y Salud Pública ${ }^{(1)}$, obteniendo en total 120 unidades bibliográficas identificadas, de las cuales un porcentaje de importancia conforma la producción médico estudiantil.

Si bien, la estrategia de búsqueda procura cubrir la alta cantidad de fuentes de información donde encontrar producción científica sanfernandina, consideramos que la estrategia de búsqueda empleada para el tópico en mención excluyó una importante cifra de investigaciones sanfernandinas al considerar solo dos eventos científicos y dos revistas científicas. Sobre los eventos científicos, si en los resultados previos se encontró un porcentaje importante de participación estudiantil, consideramos entonces pertinente la inclusión de por lo menos los libros de resúmenes de los Congresos Científicos Nacionales de Estudiantes de Medicina, organizados por la Sociedad Científica Médico Estudiantil Peruana SOCIMEP en forma anual desde hace más de 25 años ${ }^{(2)}$; donde se destaca la participación científica médico estudiantil sanfernandina (68 trabajos presentados en el período en mención, siendo 16 trabajos en educación biomédica -14 con filiación a la Sociedad Científica San Fernando), obteniéndose un indicador mayor al antes mencionado. Sobre la productividad en revistas científicas no se menciona el criterio empleado para la selección de solo dos revistas científicas biomédicas peruanas, por ello consideramos como criterio para sistematizar la selección de estas, la naturaleza de revista biomédica indizada a por lo menos la base de datos Scielo-Perú (que garantiza un proceso de revisión por pares propio de una revista científica), durante el período en mención 2005-2010, incluyendo así 10 revistas ${ }^{(3)}$ (http://www.scielo.org. $\mathrm{pe} / \mathrm{pdf} / \mathrm{afm} / \mathrm{v} 69 \mathrm{n} 1 / \mathrm{a} 09 \mathrm{v} 69 \mathrm{n} 1 . \mathrm{pdf})$, obteniendo 41 unidades bibliográficas más (tabla 1).

De esta manera podemos observar que la producción científica sanfernandina, en el período en mención, presenta mayor número de unidades bibliográficas sobre educación médi-

Tabla 1. Publicaciones sanfernandinas sobre educación médica en revistas biomédicas peruanas indexadas en Scielo-Perú 2005-2010.

\begin{tabular}{cc} 
Revista & \# artículos \\
Ciencia e Investigación Médico Estudiantil Latinoamericana (CIMEL) & $21\left(19^{*}\right)$ \\
Acta Médica Peruana & $7\left(2^{*}\right)$ \\
Revista Médica Herediana & 6 \\
Revista Peruana de Gastroenterología (RPG) & $4\left(1^{*}\right)$ \\
Folia Dermatológica Peruana & 2 \\
Dermatología Peruana & 1 \\
\hline
\end{tabular}

*Artículos con filiación Sociedad Científica San Fernando (SCSF). 
ca, y se corrobora del mismo modo la participación de la Sociedad Científica San Fernando con 63,2\% (36/57 unidades bibliográficas) de la productividad adicional hallada en esta área, siendo uno de los entes generadores de conocimiento en la facultad en este nivel.

Coincidimos con lo planteado en el artículo del Dr. Mendoza-Arana. Sin embargo, es de notar que la productividad científica en este rubro es mayor que las cifras antes mencionadas, lo cual confirma y enriquece la realidad en esta línea de investigación en nuestra facultad y de la mayor participación estudiantil que se puede observar, no solo en eventos científicos sino en las diversas revistas científicas biomédicas de nuestro medio ${ }^{(3)}$, que debería ser tomado en cuenta y permita la generación de estrategias para el mantenimiento y fortalecimiento de la investigación sanfernandina, y en particular la participación estudiantil en la facultad ${ }^{(1)}$.

\section{REFERENCIAS BIBLIOGRÁFICAS}

1. Mendoza-Arana PJ. Investigación en educación médica en la Facultad de Medicina de San Fernando: una perspectiva sistémica. An Fac med. 2012;73(1):55-61. Disponible en: http://sisbib. unmsm.edu.pe/BVrevistas/anales/v73n1/pdf/ a11v73n1.pdf
2. Huamani CA, Chávez-Solis P, Domínguez-Haro W, Solano-Aldana M. Producción científica estudiantil: Análisis y expectativas. Rev Peru Med Exp Salud Publica. 2007;24(4):444-46. Disponible en: http:// www.scielo.org.pe/pdf/rins/v24n4/a18v24n4.pdf

3. Huamani Ch, Chávez-Solis $P$, Mayta-Tristán P. Aporte estudiantil en la publicación de artículos científicos en revistas médicas indizadas en SciELO-Perú, 1997 - 2005. An Fac med. 2008;69(1):42-5. Disponible en: http://www.scielo. org.pe/pdf/afm/v69n1/a09v69n1.pdf.

\section{Conflicto de intereses:}

Los autores declaran no presentar conflicto de intereses.

Correspondencia:

e-mail corresponsal: montenegroidrogo@gmail.com

\section{LA CARTA AL EDITOR FUE REMITIDA AL AUTOR DEL ARTÍCULO EN MENCIÓN, QUIEN OFRECIÓ LA SIGUIENTE RESPUESTA.}

Lima, 29 de Agosto de 2012

Señor Doctor

José Pacheco Romero

Presidente del Comité Editorial

Anales de la Facultad de Medicina

Presente

\section{Ref: Carta al Editor de Montenegro-Idrogo, Borja-García y Gutiérrez Villafuerte}

Me dirijo a usted en relación con la Carta al Editor de la referencia, motivada por nuestro artículo sobre la investigación en educación médica en la Facultad de Medicina.

Resulta grato ver el aporte de los estudiantes de la Sociedad Científica de San Fernando a través de esta carta, la cual complementa y valida nuestros resultados. No nos cabe duda que los estudiantes son un componente esencial de la fuerza investigadora de la Facultad, y mucho más en este campo, lo cual posiblemente se relacione a que se trata de temas que los afectan de manera directa. La carta nos genera muchas inquietudes, tales como ver si las líneas de investigación elegidas por los estudiantes son coincidentes o no con las de los docentes y, en especial, si hay evidencia de un trabajo articulado o al menos coincidente en líneas temáticas. Pero, estas no son sino preguntas de investigación para continuar por este interesante campo exploratorio que nos lleve a generar recomendaciones para un desarrollo orgánico de la investigación en educación médica. Si tomamos solamente el resultado general principal de su carta, la conclusión es que los equipos de investigación estudiantiles resultan el primer generador de publicaciones en educación médica. Como docente interesado en la gestión universitaria, este solo resultado ya implica una serie de interrogantes en torno a cómo involucrar de manera orgánica este caudal, de manera que los esfuerzos investigativos estudiantiles nutran y fortalezcan los propios procesos educacionales sobre los cuales fueron aplicados.

Atentamente,

Pedro Mendoza-Arana

Profesor Principal UNMSM 